\title{
Direct measurement of muonium hyperfine splitting at J-PARC
}

\section{Sohtaro Kanda*}

The University of Tokyo

E-mail: kandadpost.kek.ip

\begin{abstract}
Muonium is a bound state of a positive muon and an electron. It is an ideal two-body system for the precision test of bound-state theory and fundamental symmetries. The ground state hyperfine splitting (HFS) of muonium provides the most rigorous test of bound-state QED theory and the most precise method to determine the muon-to-electron mass ratio. The measurement precision of the muonium HFS was mostly statistically limited because the continuous muon beam was utilized. At J-PARC, a new precision spectroscopy of the muonium HFS was performed with the high-intensity pulsed muon beam and the high-rate capable positron detector. As a result of the experiment, the muonium HFS resonance was observed and the muonium hyperfine structure interval of $\Delta v=4.463292(22) \mathrm{GHz}$ was obtained with the relative precision of $4.9 \mathrm{ppm}$. The result was consistent with the previous ones obtained at Los Alamos National Laboratory with the continuous muon beam. This article reports on the new measurement at J-PARC and its first result.
\end{abstract}

The 26th International Nuclear Physics Conference

11-16 September, 2016

Adelaide, Australia

${ }^{*}$ Speaker. 


\section{Introduction}

A hydrogen-like atom consisting of a positive muon and an electron is known as muonium. It is an ideal two-body system for the precision test of bound-state theory and fundamental symmetries. Therefore, a precision measurement of muonium ground state hyperfine splitting (HFS) is the most rigorous validation of bound-state quantum electrodynamics (QED) theory and the most precise method to determine the muon-to-electron mass ratio which is one of the essential quantities to obtain the muon anomalous magnetic moment [四]. Furthermore, a precision measurement of the muonium HFS has profound significance in search for physics beyond the standard model. As the major applications, a test for Lorentz symmetry via the measurement of sidereal oscillation [0], a search for a new massive vector boson inspired by proton radius puzzle [3], and a constraint on a long-range spin dependent interaction [䧃] are possible.

The muonium HFS was measured by two independent methods; a direct measurement at zero magnetic field and an indirect one utilizing the Zeeman splitting in a high magnetic field. The precursor experiments by both methods were performed at Los Alamos Meson Physics Facility (LAMPF) in Los Alamos National Laboratory (LANL). The most precise value of directlymeasured muonium HFS interval was $\Delta v=4.4633022(14) \mathrm{GHz}$ [5]. Note that only statistical uncertainty was considered and systematic uncertainties were not evaluated quantitatively. The measurement precision of the muonium HFS was mostly statistically limited. This article describes a new direct measurement of the muonium HFS with the high-intensity pulsed muon beam at J-PARC. The goal of the new experiment is a ten-fold of improvement in statistical precision.

\section{Experimental overview}

Figure $\mathbb{W}$ presents a schematic of the experimental apparatus. Spin polarized pulsed muon beam is irradiated to a gas chamber filled with krypton gas. A two-dimensional fiber hodoscope for muon detection is placed in front of the gas chamber to measure the profile and the intensity of the incident muon beam. An injected muon loses its kinetic energy and forms a muonium via an electron capture from krypton atom. A cylindrical microwave cavity is placed inside of the gas chamber. The cavity generates TM110 mode of a microwave resonance at the frequency of 4.463 GHz and induces the muonium HFS transition. The state transition causes a muon spin flip as a function of time. A positron is emitted by parity violating decay of muon and its emission angle is correlated to the muon spin direction. Spectroscopy of the muonium HFS is performed by a measurement of microwave frequency dependence of the number of positron from muonium decay. Positrons are detected by two layers of segmented scintillation counter with SiPM readout [四]. The detectors are placed in the downstream of the target gas chamber.

\section{Resonance spectroscopy}

Figure $\square$ shows an experimental setup of the resonance measurement. Inside of the threelayered magnetic shield, the gas chamber and particle detectors were installed. Temperature inside of the shield was controlled by a local air conditioner. Gas pressure was monitored by a capacitance gauge. Positrons from muonium decay were measured by the positron detector which was placed 


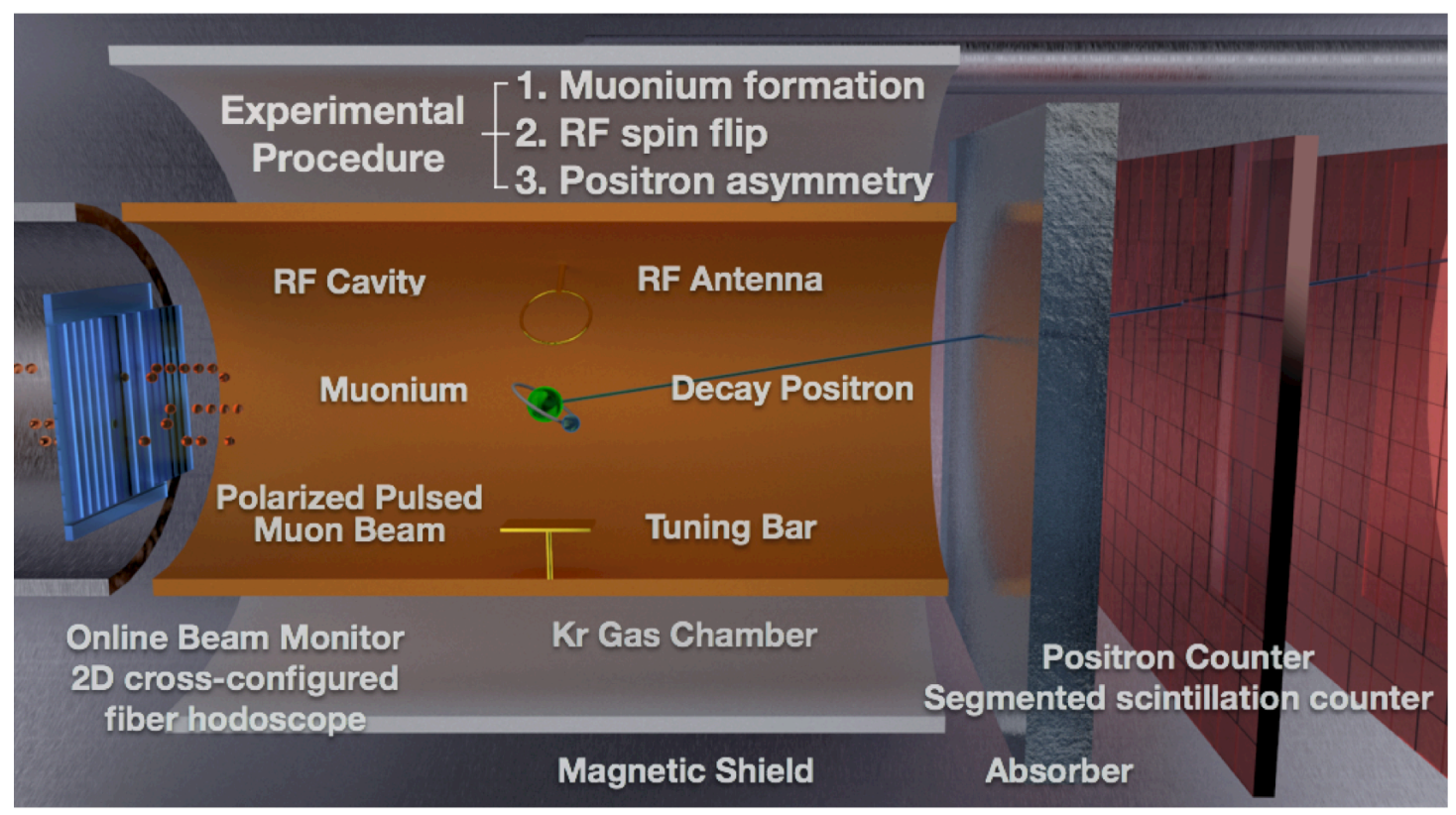

Figure 1: Experimental schematic of muonium HFS spectroscopy. Spin polarized pulsed muon beam is irradiated to $\mathrm{Kr}$ gas target. Muonium forms by an electron capture from $\mathrm{Kr}$ atom. The muonium HFS transition occurs due to the microwave resonance in the cylindrical cavity. Positrons from muonium decay are detected by the positron counter placed behind the gas chamber.

at downstream of the gas chamber. The signal from the positron detector was processed by the fast readout electronics with ASIC based amplifier, shaper, and discriminator (ASD) and multi-hit TDC implemented in FPGA. The data acquisition was triggered by the beam synchronized pulse. In order to extract a muonium spin flip signal from decay positron time spectrum, a measurement was performed both with and without microwave resonance.

Figure 3 shows the microwave cavity after installation. Magnetic loop antennas for microwave input and monitoring were installed at upper ports on the cavity. An aluminium tuning bar with piezo positioner was attached at lower port of the cavity. Position of the tuning bar was controlled remotely to adjust a resonance frequency of the microwave cavity. Microwave output was controlled remotely and switched ON/OFF every minute to prevent a local temperature rise caused by power consumption at the cavity. This microwave switching is effective to suppress the systematic uncertainties arising from the temporal variations of measurement environment. In principle, the temporal variation with a time scale of longer than minutes are highly suppressed by this technique.

\section{Data analysis}

The data analysis procedure was as follows: (1) Validation of data taking trigger by using pulse-by-pulse event rate; (2) Search for positron hit clusters and cluster merging; (3) Coincidence paring between hits on each layer of the detector; (4) Extraction of the time dependent spin flip signal by taking the counting ratio between microwave ON and OFF. 


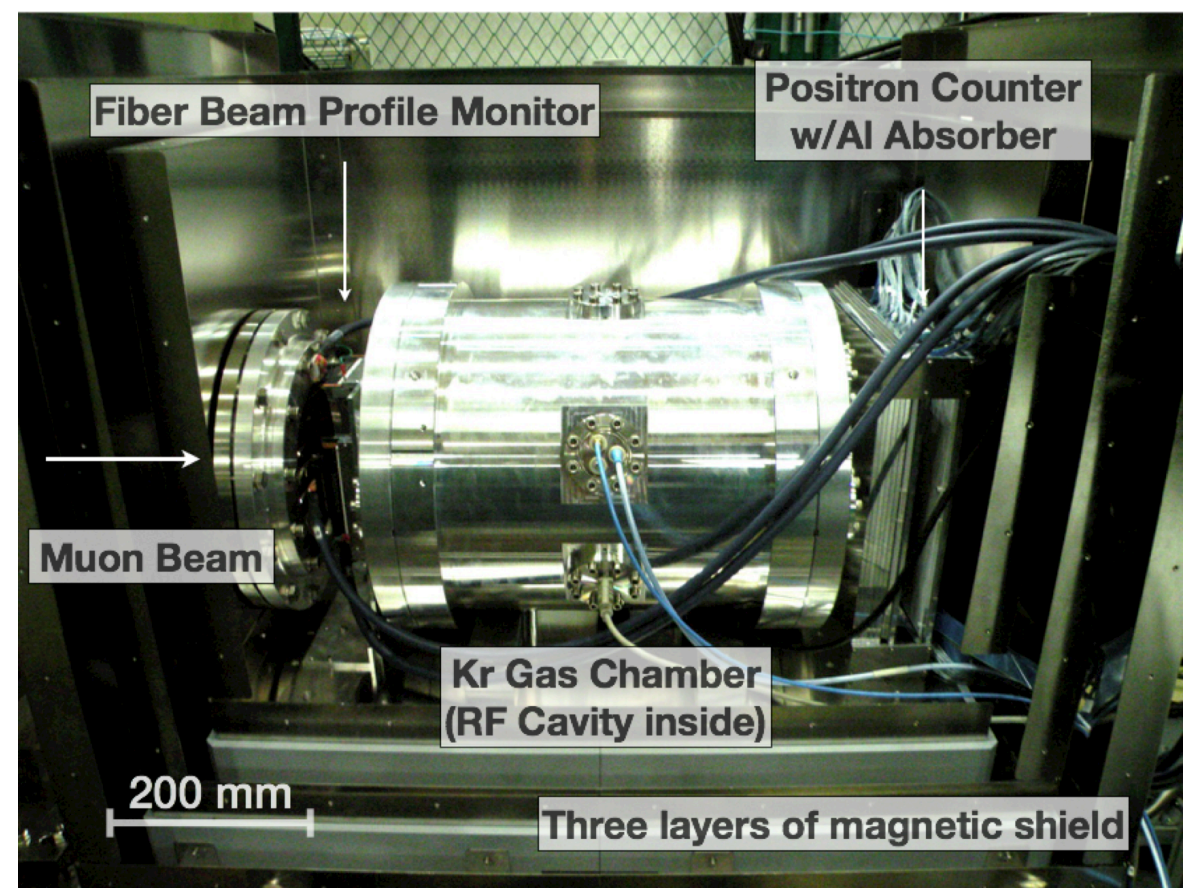

Figure 2: Experimental setup of the resonance measurement at J-PARC Material and Life Science Facility (MLF) Muon Science Establishment (MUSE). The fiber beam profile monitor and the positron detector were placed at front and behind the gas chamber, respectively. The aluminium absorber was placed between the gas chamber and the detector. During the measurement, the entire setup was enclosed by the magnetic shield.

\subsection{Time spectrum of positrons from muonium decay}

Figure 1 shows positron time spectra obtained at each step of the analysis. Each spectrum was normalized by the number of muon beam pulse. An occasional beam loss was excluded from the analysis. Cluster-like hits on a plurality of adjacent segments were observed due to an electromagnetic cascade or electrical/optical cross-talk. The hit cluster arising from these origins may cause overcounting of positrons. Therefore, the cluster hits should be searched and merged into a single aggregate. The central of each hit cluster was determined by calculation of temporal and spacial gravitational center. After a completion of hit clustering, coincidence analysis between two layers of the positron detector was performed.

\subsection{Time evolution of the muonium spin flip}

Figure $\square$ shows time dependent spin flip signals extracted from decay positron time spectra. Under an excitation of microwave resonance in the cavity, clear oscillating spin flip signal was observed. On the contrary, no significant spin flip signal was observed for a case of off-resonance tuning.

\subsection{Resonance lineshape of the muonium HFS}

As a result of decay positron counting with microwave frequency scan, a resonance lineshape of the muonium HFS transition was obtained. Figure 6 shows a measured resonance lineshape. 


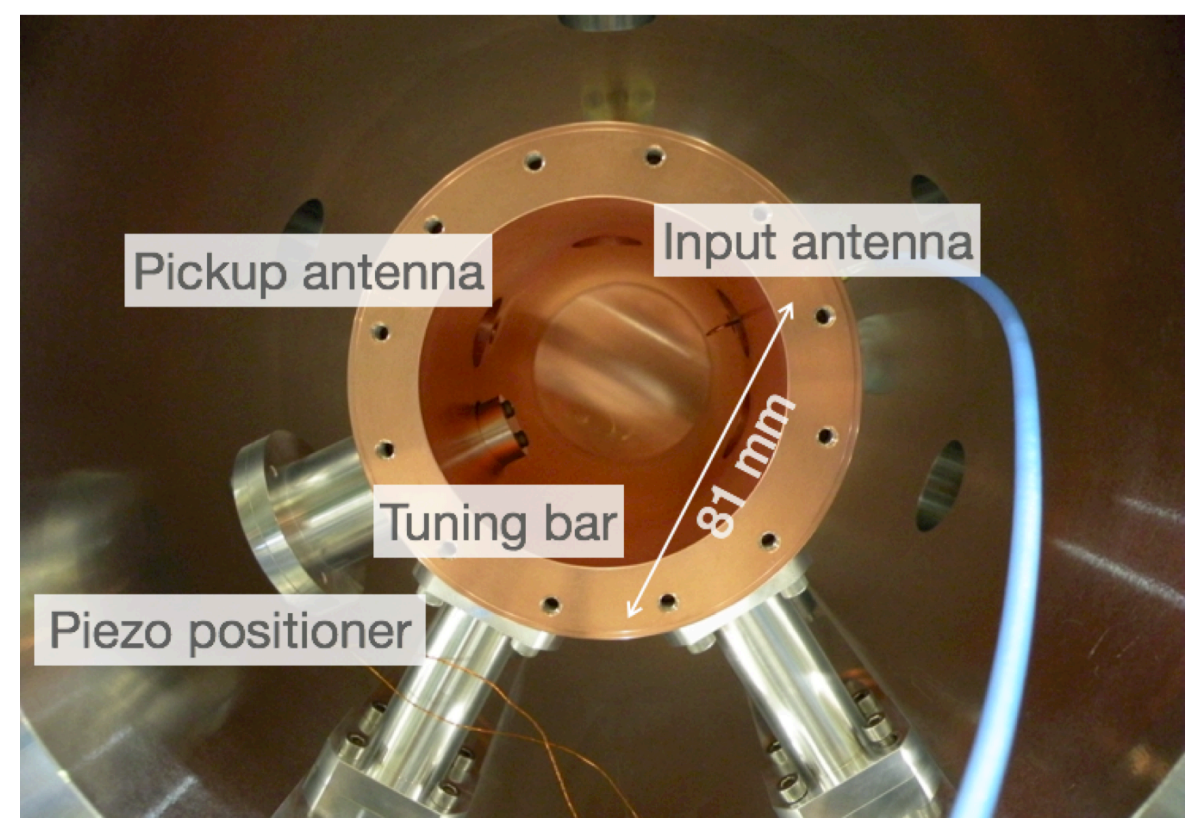

Figure 3: Microwave cavity in the gas chamber. The magnetic loop antennas for microwave input and monitoring were attached. The tuning bar with the piezo positioner was utilized for adjustment of the cavity resonance frequency.

This was the first observation of muonium HFS resonance as an experimental result with pulsed muon beam. Frequency dependence of the microwave characteristics was corrected. Fitting was performed by a theoretical expression of lineshape. For a case of wide timing range of positron integration, the function form is approximated by a Lorentzian. The obtained frequency center was $-41 \pm 22 \mathrm{kHz}$. This result corresponds to a relative uncertainty of 4.9 perts per million. An expected resonance frequency after a correction of atomic collisional shift was $-33 \mathrm{kHz}$. This result was obtained by eight-hours of measurement. The result was consistent with one by the precursor experiment. Because of a limited beam time, beam intensity, and beam-related background positrons which originate in lost muons through beam transportation, the statistical uncertainty was sixteen-fold larger than the precursor experiment result. Suppression of the beam-related background was studied and a quadruple of improvement in the precision is expected by an additional absorber for low energy positron shielding and an adaptation of a larger microwave cavity.

\section{Systematic uncertainty}

As the major contributions to systematic uncertainty, fluctuation of the microwave power and a correction of atomic collisional shift are discussed.

\subsection{Classification and evaluation of the systematics}

Systematic uncertainties are divided into two major classes. First category of interest is temporal variations of measurement condition. Both a random fluctuation and a continuous changing during the frequency scan are considered. In most cases, the continuous drift in the measurement 


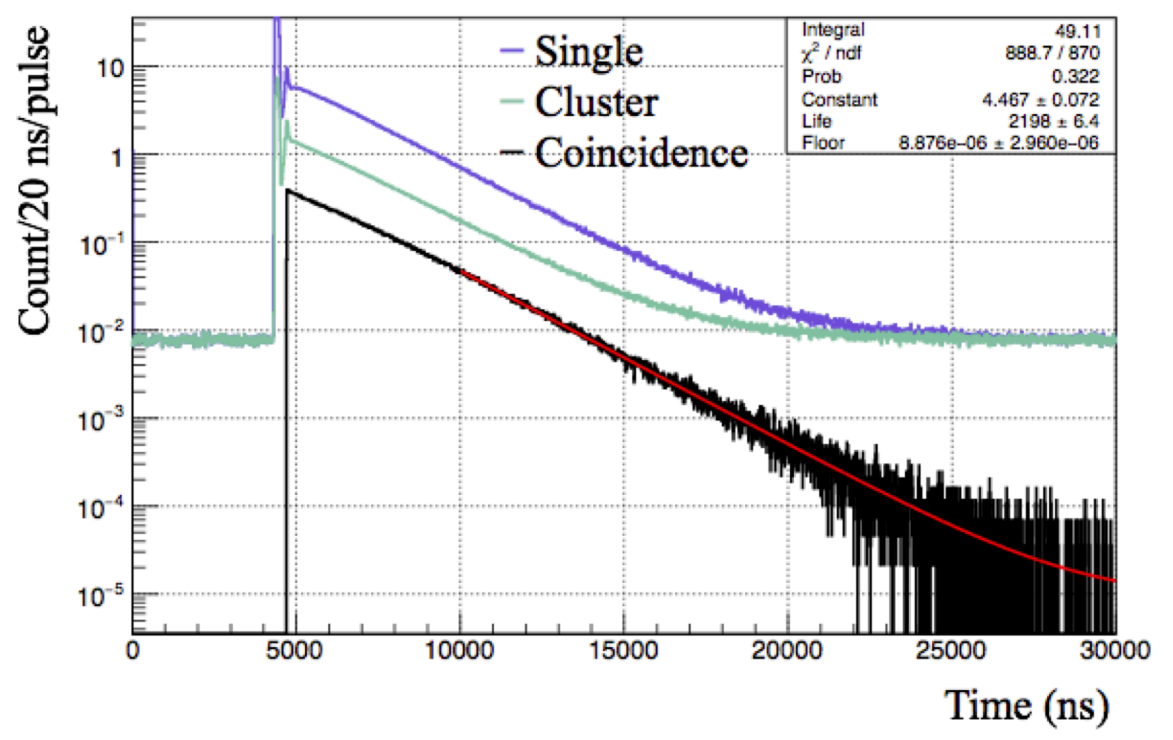

Figure 4: Time spectra of the detector hits. The histograms in blue, green, and black lines correspond to all single hits, cluster merged hits, and coincidence hits, respectively. Red line indicates the fitting result with an exponential function on a constant background.

sequence causes a systematic shift of the resonance lineshape. This type of effect can be corrected by monitoring of a correspond environmental variable. Gas pressure drift, increase of magnetic impurities in the target gas, fluctuation of muon beam intensity and profile are highly suppressed by taking the ratio of positron counting with and without microwave resonance. Only the systematic effects related to the microwave power are not suppressed by this technique. Other items are static effects arising from the remnant magnetic field, the pressure dependent atomic collisional shift, and the event loss due to the detector signal pileup. The remnant field was measured by using a fluxgate probe and the muonium HFS frequency shift due to the Zeeman effect was safely negligible. The detector pileup effect was evaluated by numerical simulation in consideration of positron event rate, photon yield, and analog circuit response.

\subsection{Microwave power stability}

Drift of the microwave power causes signal fluctuation. Stability of microwave power was monitored by using a small loop antenna attached to the microwave cavity and a thermal power sensor. During the measurement, a gradient detuning of the microwave resonance was observed. This detuning was caused by a local temperature drift on the microwave cavity. Changing in a temperature gives a slight deformation of the microwave cavity. Periodic switching of microwave output was effective to suppress the temperature drift. However, a temperature stability of the cavity was imperfect. In the data analysis, $0.5 \%$ of random fluctuation at each frequency data point was assumed based on the monitoring result. As a result of numerical calculation of the resonance lineshape with the microwave fluctuation, the systematic uncertainty in a resonance frequency was estimated to be $26 \mathrm{~Hz}$. To suppress the systematic uncertainty arising from microwave power drift, addition of feedback to the system and temperature control of the microwave cavity are effective and under study. 


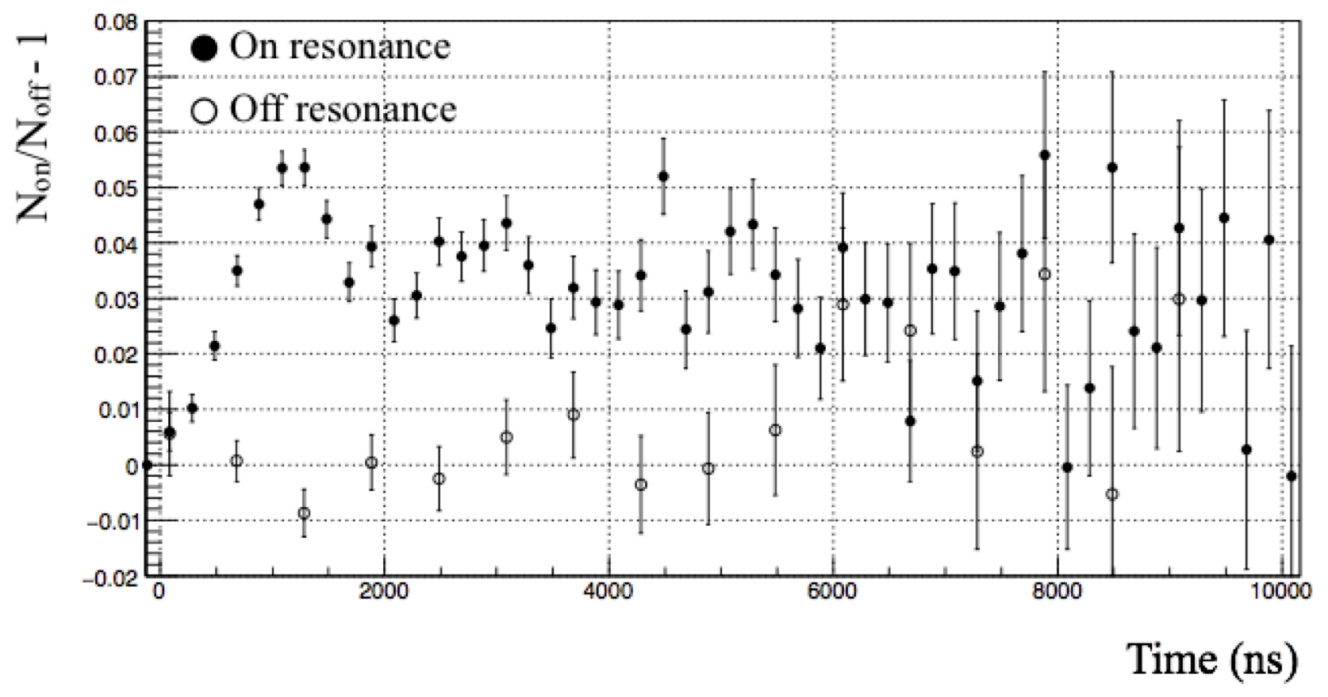

Figure 5: Time dependent signal of the muonium spin flip. Black solid circles and outlined circles correspond to the signal at on-resonance tuning and off-resonance tuning, respectively. The microwave frequency for the on-resonance measurement was tuned at $4463.3 \mathrm{MHz}$.

\subsection{Atomic collisional shift correction}

An atomic collision between muonium and krypton atom results a shift of transition frequency. This systematic effect can be evaluated as

$$
\Delta v(p)=\left(1+a p+b p^{2}\right) \Delta v(p=0)
$$

where $p$ is the gas pressure, $a$ corresponds to a contribution from two-body collision, and $b$ is a contribution from three-body collision. According to the result of precursor experiment, these coefficients were determined as follows [प]

$$
\begin{aligned}
& a=7.996(8) \times 10^{-6} / \mathrm{bar} \\
& b=5.5(1.1) \times 10^{-9} / \mathrm{bar}^{2} .
\end{aligned}
$$

For a gas pressure of $1 \mathrm{~atm}$ case, the shift of the resonance frequency is to be $33 \mathrm{kHz}$. A correction error comes from an uncertainty of the pressure gauge. In the measurement, a capacitance gauge with the precision of $0.2 \%$ was utilized. The zero-extrapolation of gas pressure arises the systematic uncertainty depends on absolute value of the gas pressure. Estimated systematic uncertainty was $66 \mathrm{~Hz}$. To suppress the systematic uncertainty origins from gas pressure extrapolation, a silicon gauge which has higher measurement precision is going to be adopted. Typical precision of a silicon gauge is $0.02 \%$, ten-fold better than a capacitance gauge. After an upgrade of a pressure gauge, uncertainties in pressure dependence parameters are to be improved. To determine gas density dependence of HFS frequency more precisely, a new measurement at several values of the gas pressure is planned. 


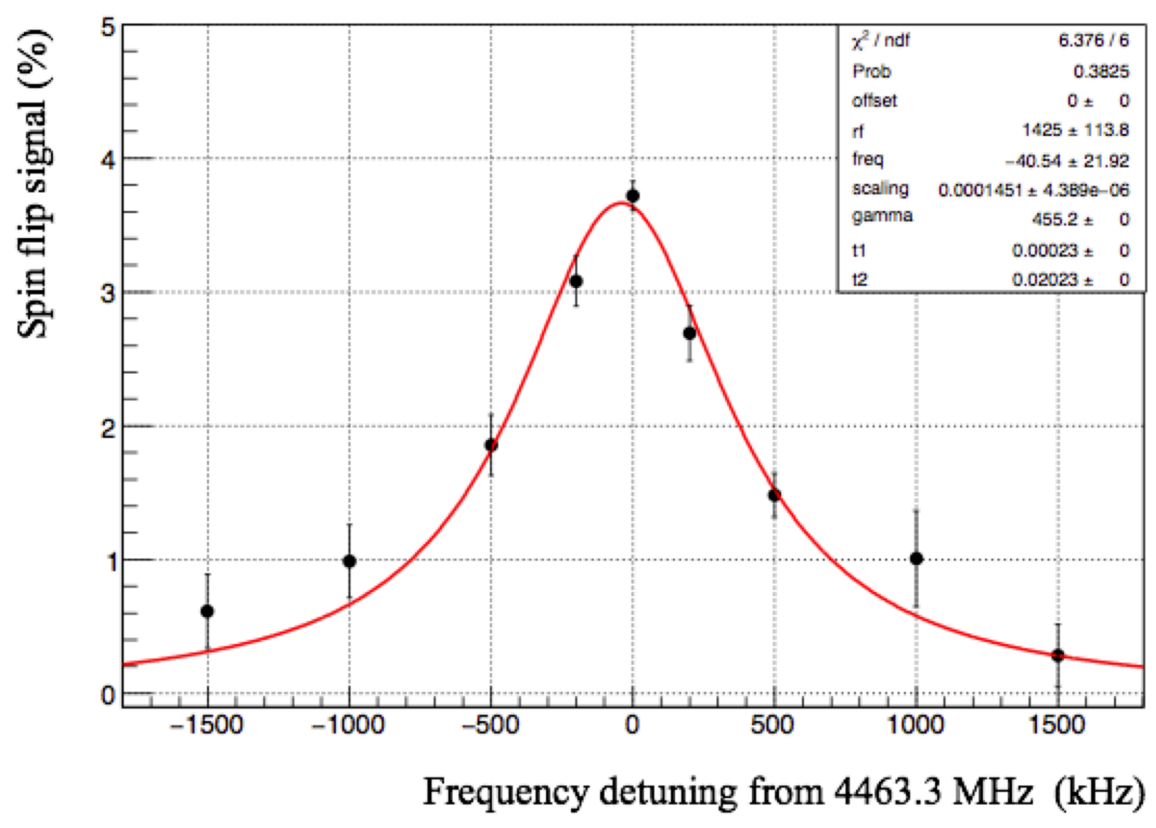

Figure 6: Resonance lineshape of the muonium HFS transition. Red line corresponds to the result of Lorentzian fitting. The vertical axis indicates the time integrated signal of the muonium spin flip. The frequency center of the resonance lineshape was evaluated to be 4463259 (22) kHz.

\subsection{Total systematics}

Estimated systematic uncertainties in the experiment were estimated as shown in Table W. With the present apparatuses, systematic uncertainty is dominated by a measurement precision of the gas pressure gauge. The second largest contribution is arising from an instability of the microwave power. For both sources of uncertainty, an order of magnitude improvements are expected by upgrades.

Table 1: Systematic uncertainties in the measurement.

\begin{tabular}{lc}
\hline Source & Contribution $(\mathrm{Hz})$ \\
\hline \hline Microwave power drift & 26 \\
Atomic collisional shift & 66 \\
Gas pressure fluctuation & 6 \\
Gas impurity & 12 \\
Muon beam intensity & 0 \\
Muon beam profile & 10 \\
Static magnetic field & 0 \\
Detector pileup & 2 \\
\hline Total & 73 \\
\hline
\end{tabular}




\section{Conclusion}

To overcome a limitation in the statistical precision achieved by the precursor experiment, the world highest-intensity pulsed muon beam was utilized. The development of the high-rate capable positron detector enabled to accept the statistical benefit of the high-intensity pulsed muon beam without increase of systematic uncertainties. Both experimental and computational approaches to the estimation of systematic uncertainties provided an understanding of the uncertainties and a suitable means to control the systematics. The magnetic shield provided a static magnetic field sufficiently close to zero from the aspect of the systematic uncertainty.

The muonium HFS resonance was successfully observed and the essential foundation of the new methodology was established. Obtained result of the muonium HFS interval was $\Delta v=$ 4.463292(22) GHz which corresponds to $4.9 \mathrm{ppm}$ of relative uncertainty.

Systematic uncertainty in the measurement was estimated to be $73 \mathrm{~Hz}$. This value is sufficiently smaller than statistical errors in both this experiment case and the precursor experiment case [ [0]. However, it is nearly equivalent to the order of total uncertainty in the most precise result obtained by an indirect measurement at a high magnetic field [8]. Towards the realization of the highest precision, room for improvement in major sources of the systematic uncertainty were studied. An order of magnitude improvement is expected by adaptation of the microwave power feedback and a precise silicon pressure gauge.

In conclusion, the experiment succeeded in the world's first demonstration of a muonium HFS spectroscopy utilizing the high-intensity pulsed muon beam.

\section{References}

[1] P. J. Mohr, D. B. Newell, and B. N. Taylor, "CODATA recommended values of the fundamental physical constants: 2014", Rev. Mod. Phys. 88, 035009 (2016).

[2] R. Bluhm, V. A. Kostelecky, and C. D. Lane, "CPT and Lorentz Tests with Muons", Phys. Rev. Lett. 84, 1098 (2000).

[3] S. G. Karshenboim, "Constraints on muon-specific dark forces", Phys. Rev. D90, 073004 (2014).

[4] S. G. Karshenboim, "Precision Physics of Simple Atoms and Constraints on a Light Boson with Ultraweak Coupling", Phys. Rev. Lett. 104, 220406 (2010).

[5] D.E. Casperson et al., "A New High Precision Measurement of the Muonium Hyperfine Structure Interval $\Delta v "$ ", Phys. Lett. (1975).

[6] S. Kanda for the MuSeUM Collaboration, Proceedings of Science, PhotoDet2015(039) (2016).

[7] P. A. Thompson, P. Crane, T. Crane, J. J. Amato, V. W. Hughes, G. zu Putlitz, and J. E. Rothberg, "Muonium. IV. Precision Measurement of the Muonium Hyperfine-Structure Interval at Weak and Very Weak Magnetic Fields", Phys. Rev. A 8, 86 (1973).

[8] W. Liu et al., "High Precision Measurements of the Ground State Hyperfine Structure Interval of Muonium and of the Muon Magnetic Moment", Phys. Rev. Lett. (1999). 